\title{
molecules
}

ISSN 1420-3049

(C) 2007 by MDPI

www.mdpi.org/molecules

Full Paper

\section{Structural Characterisation by ESI-MS of Feruloylated Arabino-oligosaccharides Synthesised by Chemoenzymatic Esterification}

\author{
Christina Vafiadi ${ }^{1}$, Evangelos Topakas ${ }^{1}$, Edwin J. Bakx ${ }^{2}$, Henk A. Schols ${ }^{2}$ and Paul \\ Christakopoulos ${ }^{1, *}$
}

${ }^{1}$ Biotechnology Laboratory, School of Chemical Engineering, National Technical University of Athens, 5 Iroon Polytechniou Str, Zografou Campus, 15700, Athens Greece

${ }^{2}$ Wageningen University, Department of Agrotechnology and Food Sciences, Laboratory of Food Chemistry,P.O. Box 8129, 6700 EV Wageningen, The Netherlands

* Author to whom correspondence should be addressed; Tel: +30-210-7723231; fax: +30-210-7723163; e-mail: hristako@orfeas.chemeng.ntua.gr

Received: 12 June 2007; in revised form: 3 July 2007 / Accepted: 3 July 2007 / Published: 9 July 2007

\begin{abstract}
The chemoenzymatic synthesis of feruloylated arabino-oligosaccharides has been achieved, using a feruloyl esterase type C from Sporotrichum thermophile (StFaeC). The structure of the feruloylated products was confirmed by ESI-MS ${ }^{\mathrm{n}}$.
\end{abstract}

Keywords: Feruloyl esterase; Enzymatic transesterification; Feruloyl oligosaccharides; Detergentless microemulsions

\section{Introduction}

Feruloyl esterases (FAEs; E.C. 3.1.1.73) are the enzymes responsible for cleaving the ester-link between the main chain polysaccharides of xylans or pectins and monomeric or dimeric ferulic acid. These polysaccharides, which complex with polymers like lignin and cellulose, modulate the plant cell wall material. FAEs are classified into four functional sub-classes (A, B, C and D) according to their primary amino acid sequence identity, specificity for the hydrolysis of hydroxycinnamic acid methyl esters, ability to release 5,5'-diferulic acid from model and complex substrates and inducible plant cell 
wall materials [1]. Various hydroxycinnamic acids (ferulic, p-coumaric, caffeic, sinapic) have widespread industrial potential by virtue of their antioxidant and antimicrobial properties [2,3]. Apart from their use as model substrates for characterisation of FAEs', methyl esters of hydroxycinnamic acids have been recently used as acid donors in transesterification reactions with aliphatic alcohols and carbohydrates [4-7].

FAEs have been used in the synthesis of sugar ferulates such as 5-O-(trans-feruloyl)- $L$-arabinofuranose [5] or $O$-[5-O-(trans-feruloyl)- $\alpha$-L-arabinofuranosyl]-(1 $\rightarrow 5)-L$-arabinofuranose [6]. More specifically, StFaeC catalyzed the transfer of the feruloyl group to $L$-arabinose and $L$-arabinobiose in a ternary water-organic mixture consisting of $n$-hexane, $t$-butanol and water, with about $40 \%$ and $20 \%$ conversion of sugar to the feruloylated derivative, respectively [7,6]. Commercial enzyme preparations from Humicola insolens, Thermomyces lanuginosus and Aspergillus niger exhibiting FAE activity were also used for the esterification of various glycosides at their primary hydroxyl group [8]. The above reactions were performed using various reaction media that enhance the solubility of the starting reagents and reverse the natural esterase hydrolytic activity to the synthetic one. Lipases are not able to catalyze such a reaction due to an electronic and/or steric effect [9].

Phenolic acid sugar esters have demonstrable antitumoric activity and have the potential to be used to formulate antimicrobial, antiviral and/or anti-inflammatory agents [10-12]. The esterification of oligo- and polysaccharides is a typical subject for both chemical and enzymatic synthesis. Production of feruloylated oligosaccharides from insoluble wheat flour arabinoxylan by treatment with a family 10 endoxylanase, showed significant antioxidant activity against 2,2-diphenyl-1-picrylhydrazyl (DPPH) reduction assay and low density lipoprotein (LDL) oxidation [13], while feruloylated oligosaccharides released from wheat bran promoted the in vitro growth of Bifidobacterium bifidum F-35 [14].

The importance of polysaccharides esterified with ferulic acid has already been reported. For example, maize starch ferulate was chemically synthesised and its food and biological properties were determined. It showed lower viscosity, higher water-holding capacity, and much less retrogradation during low temperature storage than native starch and opens another pathway to deliver health components, such as ferulic acid, safely to the colon [15]. The pasting properties using rapid viscosity analysis of commercial maize starch and starches from sorghum cultivars esterified with ferulic acid were studied, in order to investigate the model-system interactions between ferulic acid and starch. The significance of these interactions is important, especially in food matrices where phenolics are to be added as functional food ingredients [16].

In the present study, the feruloylation of four linear arabino-oligosaccharides, containing from three to six arabinofuranose units, catalysed by StFaeC, was achieved in a ternary water-organic system consisting of a mixture of $n$-hexane, $t$-butanol and water. The feruloylated oligosaccharides were detected by TLC and structurally characterized by ESI-MS ${ }^{\mathrm{n}}$ after purification.

\section{Results and Discussion}

ESI-MS ${ }^{n}$ analysis of feruloylated products

In order to determine the degree of feruloylation and the linkage position of the feruloyl group(s) on the oligosaccharide molecule and thereupon the specificity of the feruloyl esterase StFaeC in this 
kind of synthetic reaction, ESI-MS ${ }^{\mathrm{n}}$ using positive ion mode was applied. Full MSs of all products revealed the presence of mono-feruloylated oligosaccharide in each case. Full MSs of the reaction mixtures didn't show any ions corresponding to products with higher than mono- feruloylation degree in all cases (data not shown).

Figure 1. A) Second order MS of the singly charged parent ion $\left[\mathrm{M}_{1}+\mathrm{Na}\right]^{+}(\mathrm{m} / \mathrm{z} 613)$ of feruloylated arabinotriose (1). B) Third order MS of the singly charged ion $C_{2}(\mathrm{~m} / \mathrm{z} 481)$.

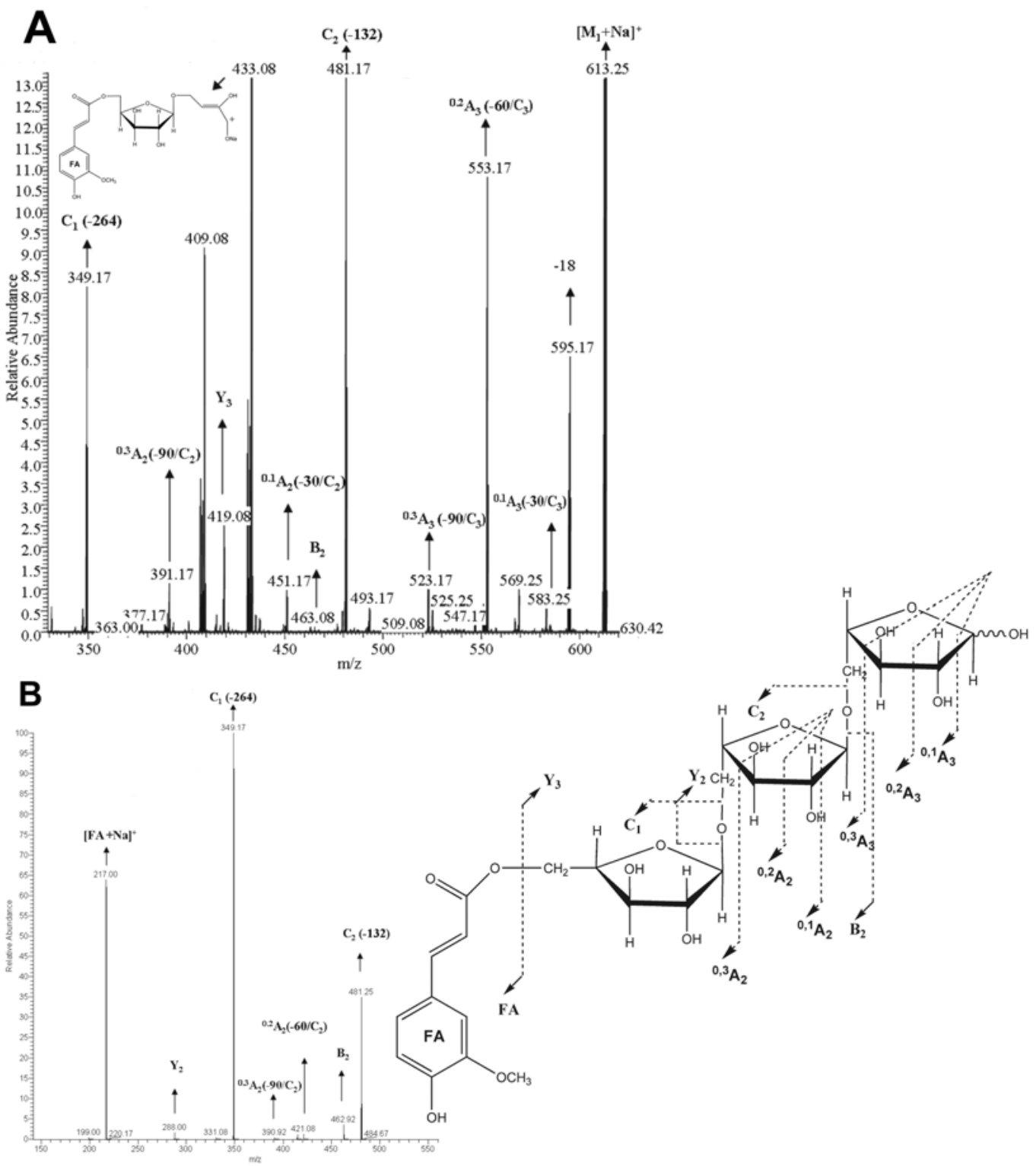

Identification of feruloylated arabinotriose $\mathbf{1}$

Second order MS of the singly charged $\left[\mathrm{M}_{1}+\mathrm{Na}\right]^{+}(\mathrm{m} / \mathrm{z}$ 613) of compound $\mathbf{1}$ is presented in Figure $1 \mathrm{~A}$. Fragments resulted from the proton rearrangement of the glycosidic bond are present, such as $\mathrm{C}_{1}$ (-264) at $m / z 349$ and $C_{2}(-132)$ at $m / z$ 481, and $\mathrm{Y}_{3}(-194)$ at $m / z 419$, which comes from the cleavage of the ester bond between ferulic acid and the oligosaccharide, accompanied by a proton transfer. 
Ions at $m / z$ 583, $m / z 553$ and $m / z 523$ correspond to the cross ring fragments ${ }^{0.1} \mathrm{~A}_{3}\left(-30 / \mathrm{C}_{3}\right),{ }^{0.2} \mathrm{~A}_{3}(-$ $\left.60 / C_{3}\right)$ and ${ }^{0.3} A_{3}\left(-90 / C_{3}\right)$ of the reducing arabinofuranose, respectively. These cross-ring fragments reveal that the reducing arabinofuranose is not esterified and that it is linked through $\mathrm{O}-5$. The same can be concluded for the Ara 2 arabinofuranose unit, because of the presence of ${ }^{0.1} A_{2}\left(-30 / C_{2}\right)$ and ${ }^{0.3} A_{2}$ $\left(-90 / C_{2}\right)$. Consequently, the ferulic acid is linked to the non-reducing end of the oligosaccharide. This is confirmed by the third order MS of the $C_{2}(m / z$ 481, Figure $1 B)$, which is consisted mainly of $C_{1}$ and the ion at $m / z 217$ which corresponds to the ferulic acid unit. Fragment $B_{2}$ at $m / z$ 463, which results from a simple cleavage of the glycosidic bond and $Y_{2}$ at $m / z$ 288, which comes from cleavage of the glycosidic bond followed by a proton transfer, are also present, together with the ring fragment at $\mathrm{m} / \mathrm{z}$ $421^{0.2} \mathrm{~A}_{2}\left(-60 / \mathrm{C}_{2}\right)$.

The fragment at $m / z 433$ shown in Figure 1A is formed after the ring cleavage predicted using the theoretical fragmentation software Mass Frontier 5.0, establishing the linkage of ferulic acid to the O-5 position of the non-reducing end of the oligosaccharide. However, the fragment at $m / z 409$ is predicted to be formed after ring cleavage of the oligosaccharide having ferulic acid esterified on the O-2 or O-3 position of the non-reducing L-arabinofuranose ring, exhibiting signal intensity higher than the signal of the O-5 fragment at $\mathrm{m} / \mathrm{z} 463$. To the best of the author's knowledge, the enzymatic esterification of the secondary alcohol group of sugars is not possible due to steric hindrance $[5,6]$, therefore this esterified product is hardly or not at all present in the reaction medium.

\section{Identification of feruloylated arabinotetraose 2}

Second order MS of the singly charged $\left[\mathrm{M}_{2}+\mathrm{Na}\right]^{+}(\mathrm{m} / \mathrm{z}$ 745) of compound 2 is presented in Figure 2A. Single feruloylation of the oligosaccharide is confirmed by the presence of $C_{1}(-396) \mathrm{at} m / z 349$, $\mathrm{C}_{2}(-264)$ at $m / z 481$ and $C_{3}(-132)$ at $m / z$ 613, as well as $Y_{4}$ at $m / z 551, B_{1}$ at $m / z 331$ and $B_{2}$ at $m / z 463$.

Cross ring fragments ${ }^{0.1} \mathrm{~A}_{3}\left(-30 / \mathrm{C}_{3}\right)$ at $\mathrm{m} / \mathrm{z} 583$ and ${ }^{0.2} \mathrm{~A}_{2}\left(-60 / \mathrm{C}_{2}\right)$ at $\mathrm{m} / \mathrm{z} 421$ indicate that the two middle arabinofuranose units $\mathrm{Ara}_{2}$ and $\mathrm{Ara}_{3}$ are not esterified. This is also confirmed by the third order MS of the $C_{3}(m / z 613$, Figure $2 B)$, where the cross ring fragments ${ }^{0.2} A_{3}\left(-30 / C_{3}\right)$ at $m / z 553$ and ${ }^{0.1} A_{2}$ $\left(-30 / C_{2}\right)$ at $m / z 451$ are present.

\section{Identification of feruloylated arabinopentaose (3) and feruloylated arabinohexaose 4}

Second order MS of compound 3 resulted in cleavage of the glycosidic bonds since the fragments $\mathrm{C}_{1}(-528)$ at $\mathrm{m} / \mathrm{z} 349, \mathrm{C}_{2}(-396)$ at $\mathrm{m} / \mathrm{z} 481$ and $\mathrm{C}_{3}(-264)$ at $\mathrm{m} / \mathrm{z} 613$ and $\mathrm{C}_{4}(-132)$ at $\mathrm{m} / \mathrm{z} 745$ were present (Figure 3). From the sequential release of mono arabinose from the reducing end without the loss of ferulic acid it can be concluded that the ferulic acid is attached to the non-reducing end of the chain. The absence of cross ring fragments obstructs the determination of the exact position of the feruloyl group on the non-reducing arabinose. The same conclusion can be drawn for the feruloylated arabinohexaose shown in Figure 4 (compound 4). 
Figure 2. A) Second order MS of the singly charged parent ion $\left[\mathrm{M}_{2}+\mathrm{Na}\right]^{+}(\mathrm{m} / \mathrm{z} 745)$ of feruloylated arabinotetraose (2). B) Third order MS of the singly charged ion $C_{3}(m / z 613)$.
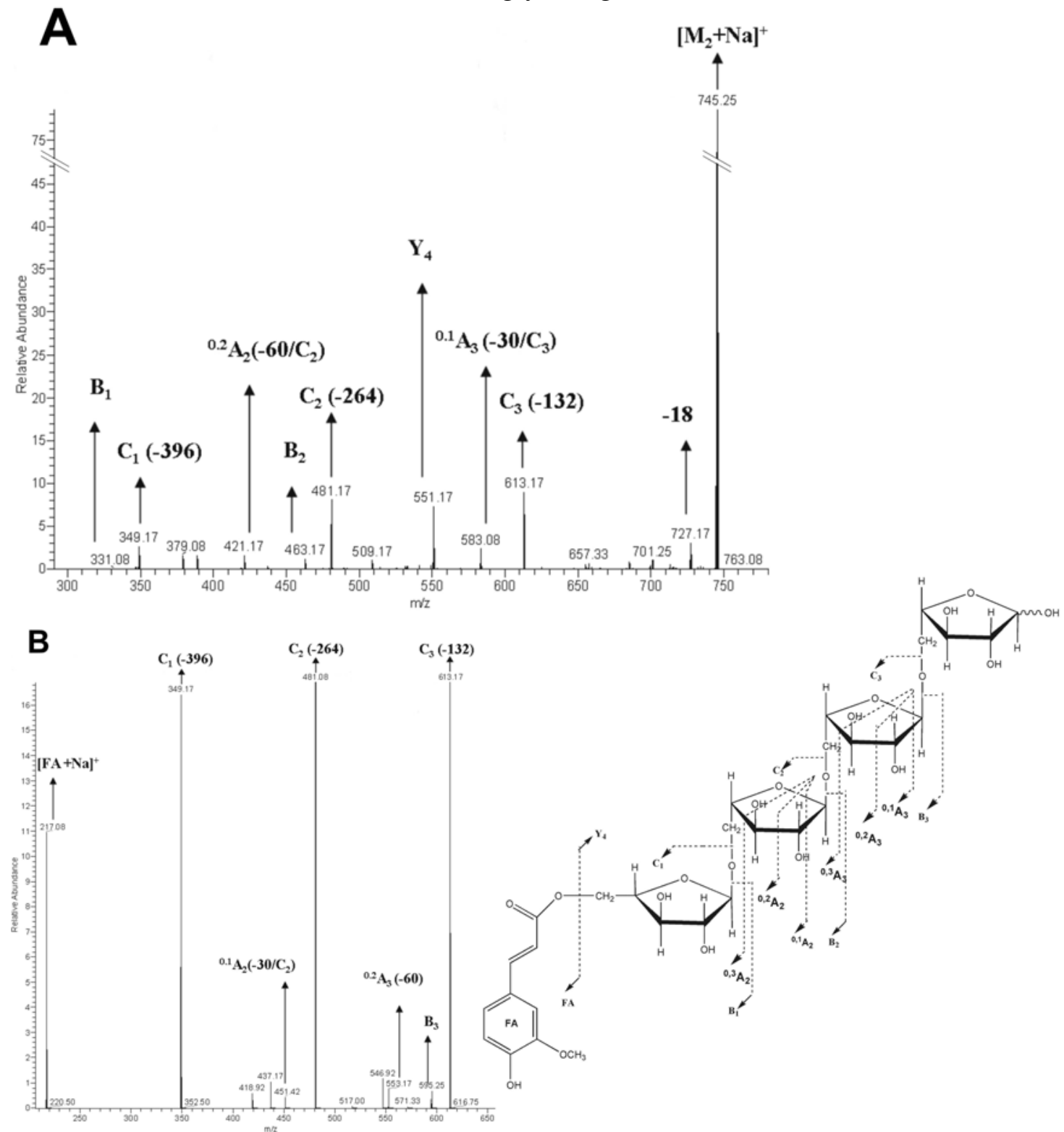

\section{Conclusions}

The present study has demonstrated the potential ability of a type C feruloyl esterase from $S$. thermophile for the feruloylation of various $L$-arabinose oligosaccharides in their non reducing arabinofuranose ring and the structural characterization of the products using ESI-MS ${ }^{\mathrm{n}}$. All the oligosaccharide substrates were mono-feruloylated as shown by the singly charged $[\mathrm{M}+\mathrm{Na}]^{+}$molecular ions of the transesterification products with ratio of $\mathrm{m} / \mathrm{z} 613$ (1),745 (2), 877 (3) and 1009 (4). As it was shown by ring fragmentation, the feruloylation took place on the non-reducing ring of each $L$ arabino-oligosaccharide studied. Moreover, these four linear oligosaccharides were possibly esterified on the primary hydroxyl group situated on the non-reducing arabinofuranose ring as reported in case of $L$-arabinose and $L$-arabinobiose transesterification reactions [7,6]. 
Further investigation should be carried out in order to expand the synthetic ability of feruloyl esterases in other reaction systems for the possible commercial exploitation of these natural modified antioxidants. The potentiality of feruloyl esterases for the esterification of polysaccharides with hydroxycinnamic acids gives a novel enzymatic approach to the synthesis of functional and biodegradable materials.

Figure 3. Second order MS of the singly charged parent ion $\left[\mathrm{M}_{3}+\mathrm{Na}\right]^{+}(\mathrm{m} / \mathrm{z}$ 877) feruloylated arabinopentaose (3)

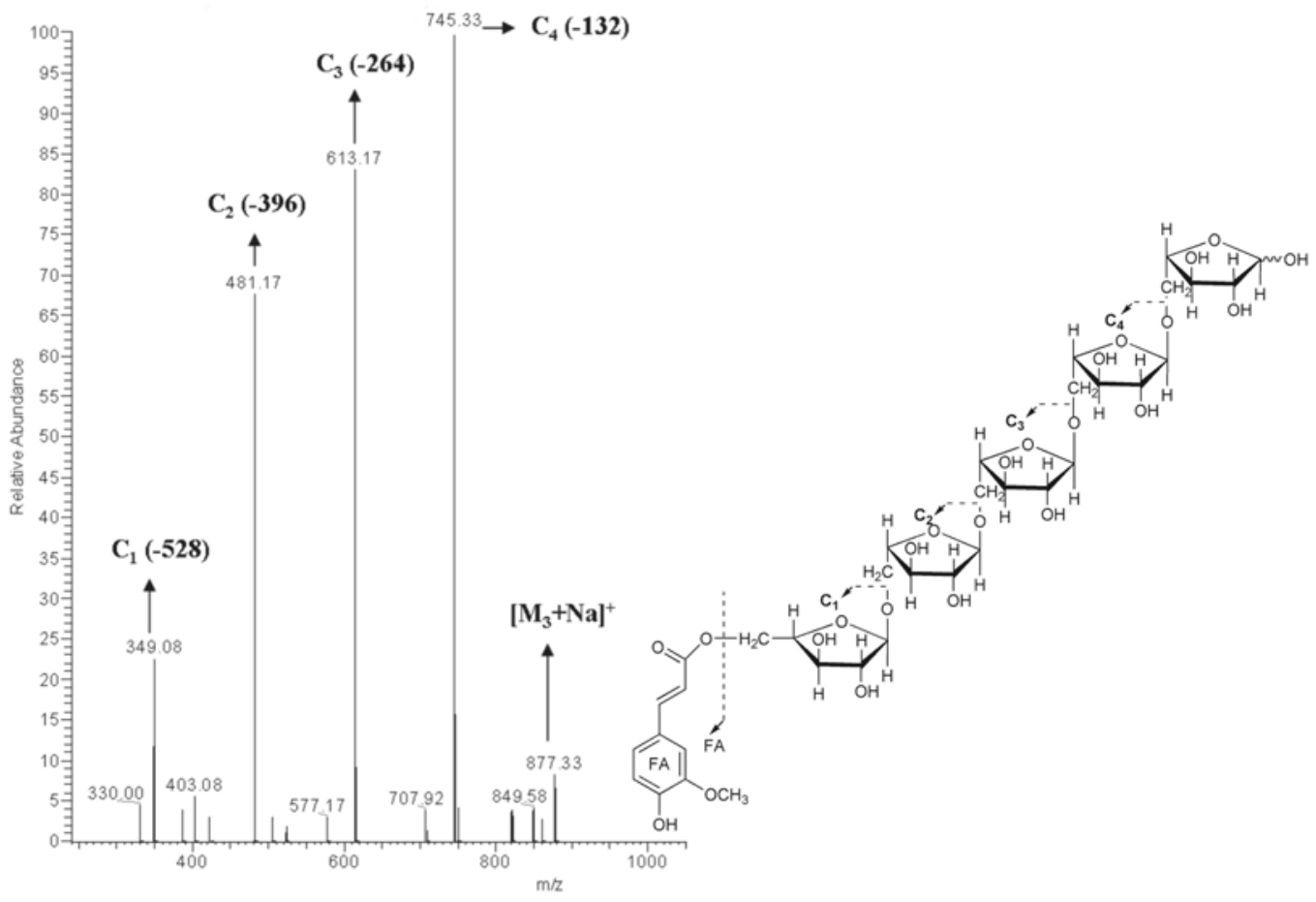

Figure 4. Second order MS of the singly charged parent ion $\left[\mathrm{M}_{4}+\mathrm{Na}\right]^{+}(\mathrm{m} / \mathrm{z} 1009)$ feruloylated arabinohexaose (4)

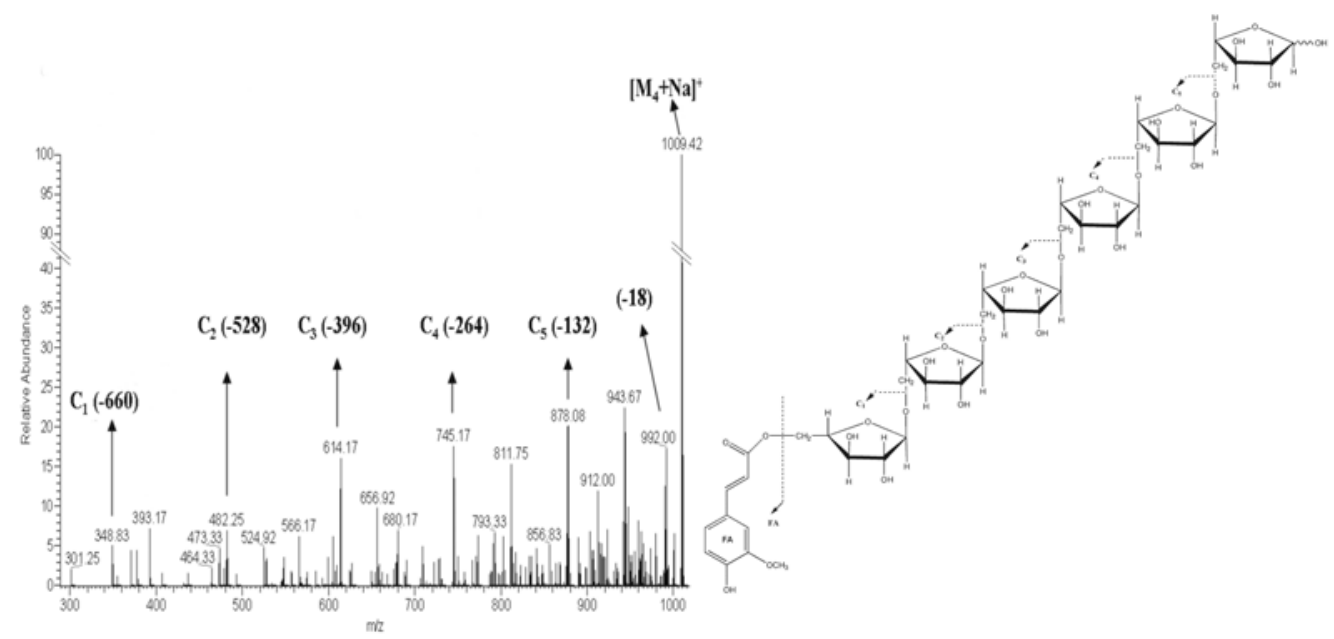




\section{Experimental}

\section{General}

The esterase described was purified to homogeneity from culture supernatants of S. thermophile grown on wheat straw, as described previously [5]. Methyl ferulate was purchased from Apin Chemicals Ltd (Abingdon, UK). Linear arabino-oligosaccharides were purchased from Megazyme (Wicklow, Ireland). All other chemicals were purchased by LabScan (Dublin, Ireland).

\section{Reactions conditions}

Surfactantless microemulsions were prepared by mixing $n$-hexane, $t$-butanol and $50 \mathrm{mM}$ buffer piperazine-HCl pH 6.0 (53.4:43.4:3.2 v/v/v), followed by vigorous shaking for several seconds until a stable transparent solution was obtained, as described previously [3]. Methyl ferulate (50 mM) was diluted in the mixture of $n$-hexane and $t$-butanol. Enzyme $(0.037 \mathrm{nM})$ and arabino-oligosaccharides (30 $\mathrm{mM}$ ) were introduced in the form of concentrated stock solution in buffer. However, sugar precipitation was observed after the addition of the concentrated stock solution into the organic solvent mixture. Enzymatic transesterification was carried out in sealed flask at $37^{\circ} \mathrm{C}$ temperature without stirring. Purification of the products was made by preparative HPLC as previously described [6].

\section{Thin Layer Chromatography}

Aliquots of reaction mixtures were spotted on aluminium sheets coated with Silicagel 60 (Merck, Germany). The solvent system that was used for the resolution of the feruloylated oligosaccharides formed was 1-butanol-acetic acid-water (50:25:25, v/v/v). TLC plates were visualized under a UV lamp (234 nm). Sugar components were detected on dried chromatograms by the aniline-hydrogen phthalate reagent.

\section{Electrospray Ionization Mass Spectrometry $\left(E S I-M S^{n}\right)$}

Electrospray ionisation mass spectrometry (ESI-MS) was performed on a LTQ Ion-trap (Thermo Electron, San Jose, USA) in positive mode using the static nanospray source with a heated capillary temperature of $200^{\circ} \mathrm{C}$ and a spray voltage of $2.2 \mathrm{kV}$. The instrument was tuned on the pentamer of glucose (present in a $10 \mu \mathrm{g} / \mathrm{mL}$ solution of maltodextrin). The sample was applied through a PicoTip emitter capillary ( $4 \mu \mathrm{m}$ ID of the tip, New Objective, Woburn, USA). MS $^{2}$ and higher were performed using a window of $1 \mathrm{~m} / \mathrm{z}$ and a relative collision energy of $30 \%$.

The Domon and Costello nomenclature [17] was used to denote the fragment ions. Fragments containing the non-reducing end are labeled ${ }^{k, l} \mathrm{~A}_{i}, \mathrm{~B}_{i}$ and $\mathrm{C}_{i}$, and those containing the reducing end are labeled $\mathrm{Y}_{i}$, where $i$ corresponds to the number of the glycosidic bond broken, counted from the non-reducing end (or the reducing end, respectively), and $k$ and $l$ indicate the cleavages within the carbohydrate ring. 


\section{Acknowledgements}

We would like to express our gratitude to the General Secretariat for Research and Technology (GSRT), Greece-Hungary bilateral program and COST D29 action for funding this work.

\section{References}

1. Crepin, V.F.; Faulds, C.B.; Connerton, I.F. Functional classification of the microbial feruloyl esterases. Appl. Microbiol. Biotechnol. 2004, 63, 647-652.

2. Shroeter, H.; Kataeva, I.A; Blum, D.L; Shah, A.K.; Ljungahl, L.G.; Rose, J.P.; Wang, B.C. Phenolic antioxidants attenuate neuronal cell death following uptake of oxidized low-density lipoprotein. Free Rad. Biol. Med. 2000, 29, 1222-1233.

3. Davidson, P.M.; Branen, A.L. Anti-microbial activity of non-halogenated phenolic compounds. J. Food. Prot. 1981, 44, 597-603.

4. Topakas, E.; Stamatis, H.; Biely, P; Kekos, D.; Macris, B.J.; Christakopoulos, P. Purification and characterization of a feruloyl esterase (FAE-II) from Fusarium oxysporum catalysing esterification of phenolic acids in ternary water-organic solvent mixtures. J. Biotechnol. 2003, 102, 33-44.

5. Topakas, E.; Vafiadi, C.; Stamatis, H.; Christakopoulos, P. Sporotrichum thermophile type C feruloyl esterase (StFaeC): Purification characterization, and its use for phenolic acid (sugar) ester synthesis. Enzyme Microb. Technol. 2005, 36, 729-736.

6. Vafiadi, C.; Topakas, E.; Christakopoulos, P. Regioselective esterase-catalyzed feruloylation of $L$ arabinobiose. Carbohydr. Res. 2006, 341, 1992-1997.

7. Vafiadi, C.; Topakas, E.; Wong, K.K.Y.; Suckling, I.D.; Christakopoulos, P. Mapping the hydrolytic and synthetic selectivity of a type C feruloyl esterase (StFaeC) from Sporotrichum thermophile using alkyl ferulates. Tetrahedron: Asymmetry 2005, 16, 373-379.

8. Mastihubova, M.; Mastihuba, V.; Bilanicova, D.; Borekova, M. Commercial enzyme preparations catalyse feruloylation of glycosides. J. Mol. Cata.l B-Enzym. 2006, 38, 54-57.

9. Figueroa-Espinoza, M-C.; Villeneuve, P. Phenolic acids enzymatic lipophilization. J. Agric. Food Chem. 2005, 53, 2779-2787.

10. Tawata, S.; Taira, S.; Kobamoto, N.; Zhu, J.; Ishihara, M.; Toyama, S. Synthesis and antifungal activity of cinnamic acid esters. Biosci. Biotechnol. Biochem. 1996, 60, 909-910.

11. Chen, X.; Martin, B.D.; Neubauer, T.K.; Linhardt, R.F.; Dordick, J.S.; Rethwisch, D.G. Enzymatic and chemoenzymatic approaches to synthesis of sugar-based polymer and hydrogels. Carbohyd. Polym. 1995, 28, 15-21.

12. Patil, N.S.; Dordich, J.S.; Retwisch, DG. Macroporous poly(sucrose acrylate) hydrogel for controlled release of macromolecules. Biomaterials 1996, 17, 2343-2350.

13. Katapodis, P.; Vardakou, M.; Kalogeris, M.; Kekos, D.; Macris, B.J.; Christakopoulos, P. Enzymic production of a feruloylated oligosaccharide with antioxidant activity from wheat flour arabinoxylan. Eur. J. Nutr. 2003, 42, 55-60.

14. Yuan, X.; Wang, J.; Yao, H. Feruloyl oligosaccharides stimulate the growth of Bifidobacterium bifidum. Anaerobe 2005, 11, 225-229. 
15. Ou, S.; Li, A.; Yang, A. A study on the synthesis of starch ferulate and its biological properties. Food Chem. 2001, 74, 91-95.

16. Corke, H.; Beta, T. Effect of ferulic acid and catechin on sorghum and maize starch pasting properties. Cereal Chem. 2004, 81, 418-422.

17. Domon, D.; Costello, C.E. A systematic nomenclature for carbohydrate fragmentations in FAB/MS/MS spectra of glycoconjugates. Glycoconjugate J. 1988, 5, 397-409.

Sample availability: Contact the authors.

(c) 2007 by MDPI (http://www.mdpi.org). Reproduction is permitted for noncommercial purposes. 